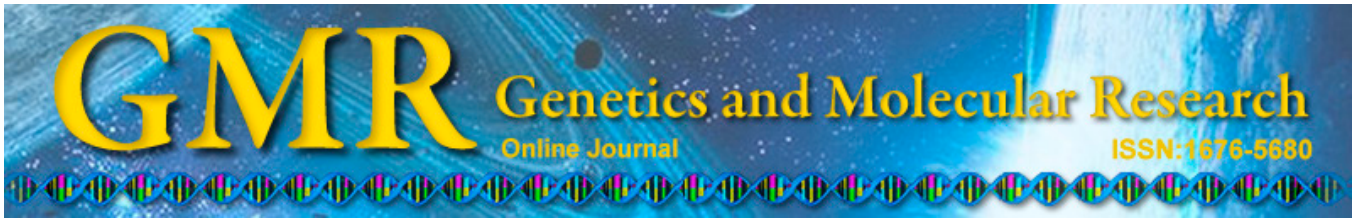

\title{
Detection of drug-resistance mechanism of Pseudomonas aeruginosa developing from a sensitive strain to a persister during carbapenem treatment
}

\author{
J.L. Shen ${ }^{1}$ and Y.P. Fang ${ }^{2}$ \\ ${ }^{1}$ Clinical Laboratory, First Affiliated Hospital of Anhui Medical University, \\ Hefei, Anhui, China \\ ${ }^{2}$ Clinical Laboratory, Second Affiliated Hospital of Anhui Medical University, \\ Hefei, Anhui, China \\ Corresponding author: J.L. Shen \\ E-mail: shenjilu_sj1@163.com
}

Genet. Mol. Res. 14 (2): 6723-6732 (2015)

Received August 15, 2014

Accepted January 22, 2015

Published June 18, 2015

DOI http://dx.doi.org/10.4238/2015.June.18.16

\begin{abstract}
We explored the mechanism of the development from sensitivity to resistance to carbapenem in Pseudomonas aeruginosa. Two $P$. aeruginosa strains were collected during treatment with carbapenem. Strain homology was investigated using pulsed-field gel electrophoresis. Porin oprD2 expression was analyzed by sodium dodecyl sulfate-polyacrylamide gel electrophoresis (SDS-PAGE). The minimum inhibitory concentrations (MICs) of imipenem and meropenem with or without MC207110 were determined using the agar dilution method. The expression level of efflux pump mRNA was tested using real-time polymerase chain reaction. Metallo-lactamases (MBLs) were screened using the EDTA-disk synergy test. Genes encoding MBLs were amplified and then analyzed by DNA sequencing. The two treated strains belonged to the same pulsed-field gel electrophoresis type. The
\end{abstract}


SDS-PAGE profile of the $P$. aeruginosa strains revealed that the $46-\mathrm{kDa}$ membrane protein OprD2 of IMP ${ }^{\mathrm{R}} \mathrm{MEM}^{\mathrm{R}}$ type strains was lost, whereas OprD2 of 1 IMPS $^{\mathrm{S}} \mathrm{MEM}^{\mathrm{S}}$ strain was normal. With or without MC207110 treatment, the MIC of carbapenem-resistant $P$. aeruginosa decreased by 4 -fold, while the MIC of carbapenem-sensitive $P$. aeruginosa did not. Compared with the carbapenem-sensitive strain, MexX mRNA expression in the carbapenem-resistant strain increased by 102.5 -fold, while the mRNA expression of other efflux pumps did not markedly increase. Neither carbapenem-resistant nor carbapenem-sensitive $P$. aeruginosa produced MBL. The mechanism of development from sensitivity to resistance of $P$. aeruginosa to carbapenem during carbapenem treatment is due to porin oprD2 loss and an increased expression level of MexXY-OprM.

Key words: Carbapenem; Carbapenemase; Efflux pumps; Porin; Pseudomonas aeruginosa; Resistance

\section{INTRODUCTION}

Carbapenem antibiotics are significant antibacterial drugs used to treat Pseudomonas aeruginosa infection. However, with the widespread use of these antibiotics, carbapenem-resis$\operatorname{tant} P$. aeruginosa strains are rapidly increasing (Wang, 2006). In clinical therapy, we found that partial $P$. aeruginosa isolates are initially sensitive and develop resistance shortly thereafter if one type of carbapenem is used. To detect the formation mechanism of carbapenem-resistant $P$. aeruginosa, we examined the mechanism of change from a sensitive strain to a persister while applying carbapenem treatment in a case of $P$. aeruginosa isolated at Huashan Hospital in 2005.

\section{MATERIAL AND METHODS}

\section{Materials}

\section{Testing strains}

Two strains of $P$. aeruginosa, including an imipenem and meropenem-sensitive strain $\left(\mathrm{IMP}^{\mathrm{S}} \mathrm{MEM}^{\mathrm{S}}\right)$ collected before treatment and an impipenem and meropenem-resistant strain $\left(\mathrm{IMP}^{\mathrm{R}} \mathrm{MEM}^{\mathrm{R}}\right)$ collected after treatment, were obtained from a patient who was infected with $P$. aeruginosa. Samples were collected before and after treatment, respectively, with carbapenems at Huashan Hospital in 2005. Two strains were identified and determined using API strips. The quality-control strain $P$. aeruginosa ATCC27853 was used in the susceptibility test, while contrast strains included standard wild strains of $P$. aeruginosa for sodium dodecyl sulfate-polyacrylamide gel electrophoresis (SDS-PAGE) analysis. The strains were provided by our institute.

\section{Antibacterials antibiotics powders}

Imipenem was provided by the Moshadong Pharmaceutical Co., Ltd. (Beijing, China) (IMP/cystatin superfamily, titer refers to IMP), piperacillin, ceftazidime, cefoperaxone, ce- 
fepime, aztreonam, amikacin, gentamicin, ciprofloxacin, meropenem, sulbactam, and tazobactam were provided by the Identification Bureau of Medicine Biological Product of Ministry of Public Health of China. These products were all standard reagents.

\section{Culture medium and biochemical reagents}

Mueller-Hinton (MH) agar medium was provided by the Oxoid (Waltham, MA, USA). Kits for extracting total RNA from bacteria were from BoDa TaiKe. The PrimeScriptTM RT Reagent Kit and SYBR Premix EX Taq were from Dalian Bao Bioengineering (Dalian, China). Tris alkali, glycine, acrylamide, N,N-acrylamide, N, N, NN-tetramethylethylenediamine, SDS, ammonium persulfate, and Coomassie brilliant blue R250 were from Xibasi Biotechnology Co. (Shanghai, China). Phosphate-buffered saline solution and destaining solution were prepared before use. Low-molecular weight standard protein (marker) was from Xibasi Biotechnology. Ethylenediaminetetraacetic acid (EDTA) $(100 \mathrm{mM}, 5 \mu \mathrm{L} / \mathrm{slice})$ and IMP/EDTA (IMP, $10 \mu \mathrm{g} / \mathrm{slice}, 0.5 \mathrm{M}$ EDTA, $\mathrm{pH} 8.0 ; 4 \mu \mathrm{L} / \mathrm{slice}$ ) solutions were prepared before use. The blank slips were provided by Tiantan Biological Products Corp. (Beijing, China). Reagents were stored at $4^{\circ} \mathrm{C}$ when necessary.

\section{Primer sequences}

The Prime Premier 5.0 software was used to design the primers, which were synthesized by Yingjun Biotechnology Co., Ltd. (Shanghai, China). The general primer name, subsequence, target gene, product longitude, and annealing temperature are shown in Table 1. The real-time polymerase chain reaction (PCR) primer subsequence was designed according to reference documents (Kolayli et al., 2004) (Table 2).

Table 1. Metal $\beta$-lactamase gene PCR amplification primer subsequence.

\begin{tabular}{|c|c|c|c|c|}
\hline Primer & Primer subsequence (location in coding gene) & Target gene & $\begin{array}{l}\text { PCR product } \\
\text { size (bp) }\end{array}$ & $\begin{array}{l}\text { Annealing } \\
\text { temperature }\end{array}$ \\
\hline VIM-2gp-A & 5'-TCCGACAGTCAYCGAAAT-3' (99-177 ) & VIM $1-6,8-12$ & 435 & $53^{\circ} \mathrm{C}$ \\
\hline VIM-2gp-B & 5'-GCAGCACCYGGATAGAAGAG-3' (514-533) & & & \\
\hline VIM-7-A & 5'-TACACCTCACCCTTGACACGC-3' (397-) & VIM 7 & 246 & $58^{\circ} \mathrm{C}$ \\
\hline VIM-7-B & 5'-ATTGGCATCGGCAACATTAC-3' (-642) & VIM-2 CDS & & \\
\hline VIMqc-A & 5'-TTGAATTCTTATGCCGCACTCACCCC-3' & & 910 & $55^{\circ} \mathrm{C}$ \\
\hline VIMqc-B & 5'-TT $\overline{\text { GGATCC }}$ AAGTTCAGCCGCCAGAA-3' & & & \\
\hline IMP-1gp-A & 5'-TCTCATTTTCATAGRGACAG-3' (280-) & IMP1, 3-7, 10 & 353 & $51^{\circ} \mathrm{C}$ \\
\hline IMP-1gp-B & 5'-ACCAGTTTTGCCTTACCATA-3' (-632) & & & \\
\hline IMP-2gp-A & 5'-CTTGTAAACACWGACGCCTAT-3' (169-) & IMP2, $8,13,19,20$ & 134 & $52^{\circ} \mathrm{C}$ \\
\hline IMP-2gp-B & 5'-GTGCTGTCGCTATGGAAAT-3' (-302) & & & \\
\hline IMP-11gp-A & 5'-GKGTCTTTGCCTGATTTA-3' (58-) & IMP11, 16, 21 & 256 & $51^{\circ} \mathrm{C}$ \\
\hline IMP-11gp-B & 5'-CTATTCCACCCGWGCTGT-3' (-313) & & & \\
\hline IMP-14gp-A & 5'-RGACAGTACGGCTGGAATAG-3' (294-) & IMP14. 18 & 239 & $55^{\circ} \mathrm{C}$ \\
\hline IMP-14gp-B & 5'-CAAAGCAACCACCGAATAAA-3' (-532) & & & \\
\hline IMP-12gp-A & 5'-TTRCATAGCGACAGRACG-3' (286-) & IMP9, 12,15 & 277 & $52^{\circ} \mathrm{C}$ \\
\hline IMP-12gp-B & 5'-CARCCAAATTACCWAGACC-3' (-562) & & & \\
\hline IMP-2qc-A & 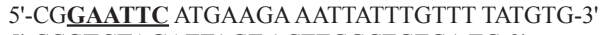 & IMP-2 CDS & 741 & $55^{\circ} \mathrm{C}$ \\
\hline IMP-2qc-B & 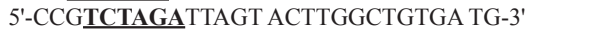 & & & \\
\hline
\end{tabular}


Table 2. Real-time PCR primer sequence.

\begin{tabular}{|c|c|c|}
\hline Primer & Primer subsequence ( $5^{\prime}$ to $\left.3^{\prime}\right)$ & Reference documents \\
\hline rpoD-F & GGGCGAAGAAGGAAATGGTC & Kolayli et al. \\
\hline rpoD-R & CAGGTGGCGTAGGTGGAGAA & \\
\hline oprD-F & TCCGCAGGTAGCACTCAGTTC & Kolayli et al. \\
\hline oprD-R & AAGCCGGATTCATAGGTGGTG & \\
\hline mexA-F & AACCCGAACAACGAGCTG & Kolayli et al. \\
\hline mexA-R & ATGGCCTTCTGCTTGACG & \\
\hline mexC-F & GGAAGAGCGACAGGAGGC & Quale et al. \\
\hline mexC-R & CTGCACCGTCAGGCCCTC & \\
\hline mexE-F & TACTGGTCCTGAGCGCCT & Quale et al. \\
\hline mexE-R & TCAGCGGTTGTTCGATGA & \\
\hline $\operatorname{mexX}-\mathrm{F}$ & GGCTTGGTGGAAGACGTG & Quale et al. \\
\hline mexX-R & GGCTGATGATCCAGTCGC & \\
\hline
\end{tabular}

\section{Methods}

\section{Drug susceptibility testing}

The minimal inhibitory concentration (MIC) testing to IMP and MEM and other antibiotics were used according to agar 2-fold dilution as recommended by Clinical and Laboratory Standards Institute in 2005.

\section{Multidrug excretion pump phenotype testing}

We tested the MIC of single IMP and MEM application in agar dilution, as well as with 2 sets of the inhibitor MC207110, which was added to a final concentration of $20 \mathrm{mg} /$ $\mathrm{mL}$. The concentration range of IMP and MEM was $0.06-128 \mathrm{mg} / \mathrm{mL}$. The excretion pump phenotype was determined; $P$. aeruginosa can be considered to have an excretion pump mechanism of resistance if the MIC decreases by 4-fold or more while adding MC207110 (Xu et al., 2007).

\section{Pulsed-field gel electrophoresis (PFGE) using homologization analysis}

The 2 strains were analyzed using the protocols described by the United States Centers for Disease Control and Prevention. Digestion with the restriction enzyme SpeI produced 20-25 fragments ranging in size from 10 to $700 \mathrm{~kb}$. PFGE results were analyzed using homologization evaluation criteria (Tenover et al., 1995).

\section{Metal-lactamase detection}

For the EDTA synergy test, we used the method described by Luo et al. (2007). Strains were cultured on MH plates to a turbidity of $0.5 \mathrm{McF}$ arland standard, and then IMP sticks were added. First, the blank slip paper was spread $(10 \mathrm{~g})$ near the IPM stick, and then dropped into $4 \mu \mathrm{L} 0.5 \mathrm{M}$ EDTA. The mixture was incubated overnight at $35^{\circ} \mathrm{C}$. IMP inhibition zones that appear approaching the EDTA paper represent a "spoon hole" phenomenon. Obviously large inhibition zones indicated metal lactamase-positive strains. 


\section{Membrane protein content and analysis}

Membrane protein content was evaluated according to previously described methods (Chen et al., 2001). A BCA kit was used to determine protein concentration, which was then adjusted to $40 \mathrm{~g} / \mathrm{L}$. After electrophoresis, the gel was stained for more than $4 \mathrm{~h}$ with Coomassie bright blue. After 4-8 h destaining, photos were acquired using a Bio-Rad GelDoc XR gelatin imaging system (Bio-Rad, Hercules, CA, USA).

\section{Real-time PCR test for oprD2 and excretion pump gene mRNA expression levels}

Real-time PCR was used to test oprD2 and excretion pump (MexA, MexC, MexE, MexX) mRNA expression levels according to a previously described method (Masuda et al., 1995). Total RNA was extracted and genomic DNA was digested. Products were amplified and purified by Sepharose electrophoresis in order to determine whether primer dimers were present. The housekeeping gene rpoD was used as an internal reference. Relative gene expression was evaluated using the CT method (Pfaffl, 2001).

\section{RESULTS}

\section{Example source and case feature}

The male patient, aged 98 years, was admitted on October 1, 2000 to the Senion Officials Inpatient Ward because of brain infarction. From then on, this patient had recurrent pulmonary infection and $P$. aeruginosa was isolated many times from sputum incubation. Separation time, primary diseases, antibiotics status during therapy, and the two $P$. aeruginosa strains isolated from the patient are shown in Table 3.

$\begin{aligned} & \text { Table 3. General situation, primary diseases, antibiotics employed status, and prognosis of Pseudomonas } \\
& \text { aeruginosa } \text { patient. }\end{aligned}$
\begin{tabular}{lcllll}
\hline Strains & Separation time & Diagnosis & Basis disease & Antibiotics-employed status & Prognosis \\
\hline IMPS $^{\mathrm{S}} \mathrm{MM}^{\mathrm{S}}$ & $2005-5-20$ & $\begin{array}{l}\text { Pulmonary } \\
\text { infection }\end{array}$ & $\begin{array}{l}\text { Cerebral infarction, } \\
\text { brain atrophy, coronary } \\
\text { artery disease }\end{array}$ & Imipenem & Not improved \\
IMP $^{\mathrm{R}} \mathrm{MEM}^{\mathrm{R}}$ & $2005-6-4$ & & Levofloxacin + imipenem & Improved after treatment \\
\hline
\end{tabular}

\section{Antimicrobial susceptibility tests}

The results of antimicrobial susceptibility tests for the 2 strains of $P$. aeruginosa showed that the MIC of the strain susceptible to IMP and MEM was $4 \mathrm{mg} / \mathrm{L}$. The MICs of the strain resistant to IMP and MEM were 16 and $64 \mathrm{mg} / \mathrm{L}$. Both the susceptible and resistant strains were sensitive to polymyxin. The MICs of the two P. aeruginosa strains to other antimicrobial agents are shown in Table 4. 
Table 4. Sensitivity of Pseudomonas aeruginosa strains $\mathrm{IMP}^{\mathrm{S}} \mathrm{MEM}^{\mathrm{S}}$ and $\mathrm{IMP}^{\mathrm{R}} \mathrm{MEM}^{\mathrm{R}}$ to antimicrobial agents $(\mathrm{MIC}, \mathrm{mg} / \mathrm{L})$.

\begin{tabular}{|c|c|c|c|c|c|}
\hline Antimicrobial agents & $\mathrm{IMP}^{\mathrm{S}} \mathrm{MEM}^{\mathrm{S}}$ & IMPR $^{\mathrm{R}} \mathrm{MEM}^{\mathrm{R}}$ & Antimicrobial agents & $\mathrm{IMP}^{\mathrm{S}} \mathrm{MEM}^{\mathrm{S}}$ & $\mathrm{IMP}^{\mathrm{R}} \mathrm{MEM}^{\mathrm{R}}$ \\
\hline Piperacillin & S (32) & S (32) & Ciprofloxacin & $\mathrm{R}(16)$ & $\mathrm{R}(32)$ \\
\hline Ceftazidime & $\mathrm{S}(8)$ & $\mathrm{S}(8)$ & Imipenem & S (4) & $\mathrm{R}(16)$ \\
\hline Cefoperazone & $\mathrm{R}(128)$ & $\mathrm{R}(128)$ & Meropenem & S (4) & $\mathrm{R}(64)$ \\
\hline Cefepime & $\mathrm{R}(32)$ & $\mathrm{R}(32)$ & Piperacillin/tazobactam & $S(32)$ & $S(32)$ \\
\hline Aztreonam & $\mathrm{R}(32)$ & $\mathrm{R}(32)$ & Cefoperazone/sulbactam & $\mathrm{R}(128)$ & $\mathrm{R}(128)$ \\
\hline Amikacin & S (16) & S (16) & Polymyxin & $\mathrm{S}(2)$ & $\mathrm{S}(2)$ \\
\hline Gentamicin & $\mathrm{I}(8)$ & $\mathrm{I}(8)$ & & & \\
\hline
\end{tabular}

S: sensitive; I: mediation; R: drug resistance.

\section{Results of PFGE homologization analysis}

The PFGE graph of the two isolated P. aeruginosa strains was analyzed according to Tenover et al. (1995). The PFGE bands of the two strains showed the same spectral pattern and were not distinct (Figure 1).

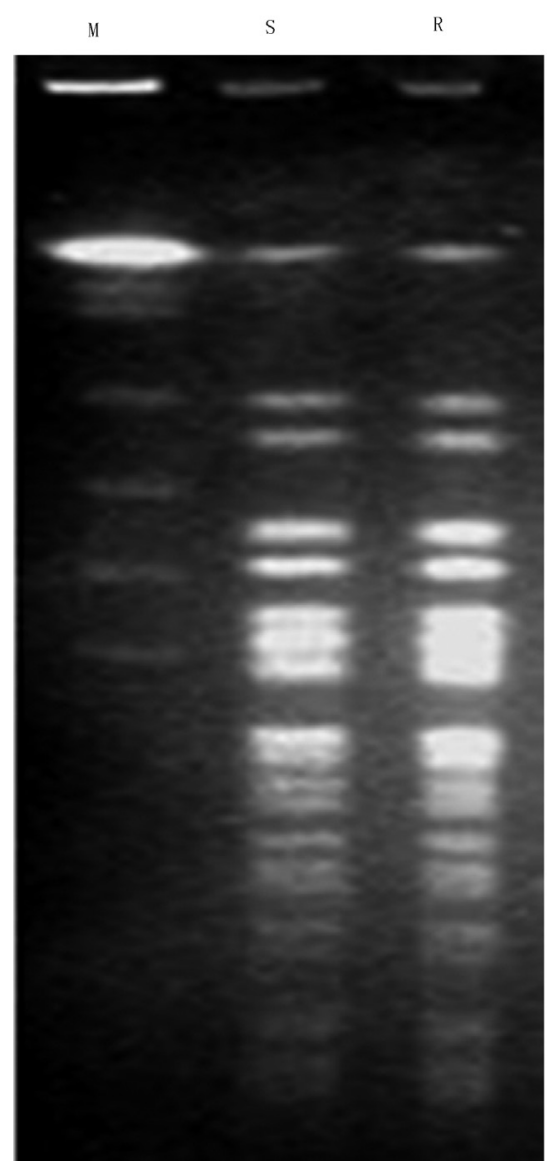

Figure 1. PFGE results of Pseudomonas aeruginosa $\mathrm{IMP}^{\mathrm{S}} \mathrm{MEM}^{\mathrm{S}}$ and $\mathrm{IMP}^{\mathrm{R}} \mathrm{MEM}^{\mathrm{R}}$. (lane M: marker; lane $S$ : $\mathrm{IMP}^{\mathrm{S}} \mathrm{MEM}^{\mathrm{S}}$ strain; lane $R$ : $\mathrm{IMP}^{\mathrm{R}} \mathrm{MEM}^{\mathrm{R}}$ strain). 


\section{Metallo-lactamase phenotype screening tests and PCR detection of -lactamase gene}

The metal-lactamase phenotype screening tests and PCR detection of the -lactamase gene for the two $P$. aeruginosa strains showed that carbapenem detection was negative.

\section{Membrane protein analysis results}

The SDS-PAGE results showed that the carbapenem-sensitive strain obtained from the patient before clinical treatment showed a band at $46 \mathrm{kDa}$ for the oprD protein, but after treatment, the acquired persister did not show a band at $46 \mathrm{kDa}$ (Figure 2).

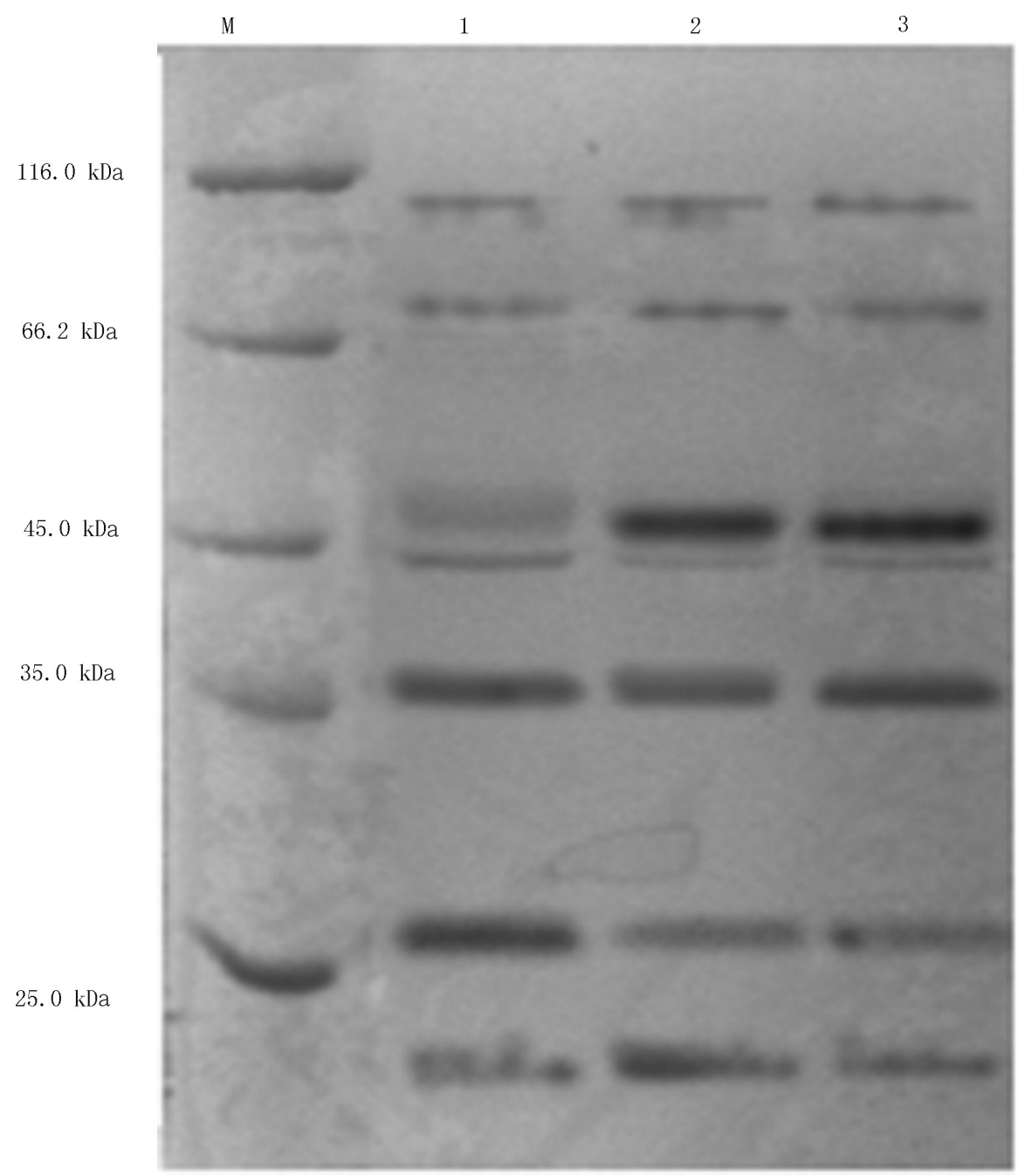

Figure 2. Results of IMPS MEM ${ }^{\mathrm{S}}$ and $\mathrm{IMP}^{\mathrm{R}} \mathrm{MEM}^{\mathrm{R}}$ strains of Pseudomonas aeruginosa in outer membrane protein SDS-PAGE electrophoresis. Lane $M$ : standard protein marker; lane 1: $\mathrm{IPM}^{\mathrm{R}} \mathrm{MEM}^{\mathrm{R}}$ strain; lane 2: $\mathrm{IPM}^{\mathrm{S}} \mathrm{MEM}^{\mathrm{S}}$ strain; lane 3: PAO1 strain. 


\section{Phenotypic detection of efflux pump}

We determined the sensitivity of the two P. aeruginosa strains to IMP and MEM using the pump inhibitor MC207110. The results showed that the MIC of the IMPS $\mathrm{MEM}^{\mathrm{S}}$ strain following single IMP and MEM and combined treatment with pump inhibitor was respectively 4 $\mathrm{mg} / \mathrm{L}$. The MICs of IMP ${ }^{\mathrm{R}} \mathrm{MEM}^{\mathrm{R}}$ following single IMP and MEM treatments were 16 and 64 $\mathrm{mg} / \mathrm{L}$, respectively. When combined with pump inhibitor, the MIC of MEM in the IMPS MEM ${ }^{\mathrm{s}}$ strain was decreased by 4 -fold. The MIC of IMP did not show a clear decrease (Table 5).

Table 5. MIC of single sensitive strain and single resistant strain combined with MC207110 (mg/L).

\begin{tabular}{lcccr}
\hline Strains & \multicolumn{2}{c}{ Single medicine } & \multicolumn{2}{c}{ Combined with MC207110 } \\
\cline { 2 - 4 } & Imipenem & Meropenem nnem & Imipenem & 4 \\
\hline IMPS $^{\mathrm{S}}$ MEM $^{\mathrm{S}}$ & 4 & 4 & 4 & Meropenem \\
IMP $^{\mathrm{R}} \mathrm{MEM}^{\mathrm{R}}$ & 16 & 64 & 8 & 16 \\
\hline
\end{tabular}

\section{Outer membrane protein oprD2 and excretion pump gene mRNA expression}

The mRNA expression levels of the outer membrane protein oprD2 and excretion pump genes (MexA, MexC, MexE, MexX) were examined using real-time PCR detection. The results showed that the mRNA expression level of the MexX gene was increased by 102.5-fold in the two P. aeruginosa strains. Changes in the mRNA expression level of the other genes (MexA, MexC, MexE) were not clear. The mRNA expression level of the resistant strain showed some changes, but these changes were not as clear as those observed in the sensitive strain (Table 6).

Table 6. Detection of Pseudomonas aeruginosa excretion pump gene and mRNA expression level.

\begin{tabular}{lcccrcr}
\hline Strains & Gene name & Average $\mathrm{C}_{\mathrm{T}}$ & $\begin{array}{c}\text { Interior reference } \\
\text { average rpo } \Delta \mathrm{C}_{\mathrm{T}}\end{array}$ & $\Delta \mathrm{C}_{\mathrm{T}}$ & $\begin{array}{c}\Delta \Delta \mathrm{C}_{\mathrm{T}} \\
\text { Average relative } \\
\text { content }\end{array}$ \\
\hline Sensitive strain & oprD & $17.25 \pm 0.43$ & $17.31 \pm 0.23$ & $-0.06 \pm 0.49$ & $0.00 \pm 0.49$ & 1 \\
Resistant strain & & $15.20 \pm 0.06$ & $15.50 \pm 0.04$ & $0.3 \pm 0.07$ & $-0.24 \pm 0.07$ & 1.2 \\
Sensitive strain & mexA & $17.73 \pm 0.11$ & $17.31 \pm 0.23$ & $0.42 \pm 0.25$ & $0.00 \pm 0.25$ & 1.0 \\
Resistant strain & & $15.85 \pm 0.13$ & $15.50 \pm 0.04$ & $0.35 \pm 0.14$ & $-0.07 \pm 0.14$ & 1.0 \\
Sensitive strain & \multirow{2}{*}{ mexC } & $21.99 \pm 0.26$ & $17.31 \pm 0.23$ & $4.68 \pm 0.35$ & $0.00 \pm 0.35$ & 1 \\
Resistant strain & & $18.44 \pm 0.14$ & $15.50 \pm 0.04$ & $2.94 \pm 0.15$ & $-1.74 \pm 0.15$ & 3.3 \\
Sensitive strain & \multirow{2}{*}{ mexE } & $21.00 \pm 0.31$ & $17.31 \pm 0.23$ & $3.69 \pm 0.38$ & $0.00 \pm 0.38$ & 1 \\
Resistant strain & & $17.58 \pm 0.14$ & $15.50 \pm 0.04$ & $2.08 \pm 0.15$ & $-1.61 \pm 0.15$ & 3.0 \\
Sensitive strain & \multirow{2}{*}{ mexX } & $27.11 \pm 0.49$ & $17.31 \pm 0.23$ & $9.80 \pm 0.54$ & $0.00 \pm 0.54$ & 1 \\
Resistant strain & & $18.62 \pm 0.67$ & $15.50 \pm 0.04$ & $3.12 \pm 0.67$ & $-6.68 \pm 0.67$ & 102.5 \\
\hline
\end{tabular}

\section{DISCUSSION}

P. aeruginosa often causes infection in patients showing low immunity (Masuda et al., $1995)$ and is a significant conditional pathogenic bacteria in clinic practice. P. aeruginosa presents intrinsic and acquired multidrug resistance to widely used antimicrobials. Once infected, therapy is difficult. We often find that $P$. aeruginosa is sensitive to carbapenem during the initial stage of treatment, but the bacteria may rapidly develop resistance to this agent during treatment.

In this study, we conducted a susceptibility test for IMP and MEM in two P. aerugi- 
nosa strains isolated from a patient before and after treatment, respectively. The PFGE results revealed that the two strains were from the same clone. We observed three main differences between the resistant strain obtained after treatment and the sensitive strain obtained before treatment, enabling study of the $P$. aeruginosa resistance mechanism.

The resistant strain lost the oprD2 protein, while the sensitive strain did not. After combined treatment with MC207110 and MEM, the MIC of the resistant strain decreased by 4-fold, while no change was observed in the sensitive strain. The mRNA expression level of the MexX gene, belonging to the multidrug excretion system MexXY-oprM, was increased by 102.5-fold compared to the sensitive strain. EDTA synergism testing and PCR detection of metal-lactamase were negative. This indicates that the formation of resistance to IMP and MEM may have decreased the membrane protein oprD2 expression, while the excretion pump MexXY-OprM system was increased; this pump participates in the development of $P$. aeruginosa resistance to IMP and MEM rather than development of carbapenem resistance.

As reported previously (Yoneda et al., 2005), because of the post-transcriptional control mechanism, the expression level of OprD2 protein is inconsistent with its mRNA levels, and the protein level can be low while the mRNA level is normal. Additional studies are needed to determine the cause of this inconsistency.

Although multiple excretion systems have been reported for $P$. aeruginosa, only MexAB-OprM (Poole et al., 1993), MexEF-OprN (Kohler et al., 1997), and MexXY-OprM (Mine et al., 1999) are related to the formation of carbapenem-resistance and FQs are substrates of these excretion pump systems. FQs can induce excretion pump system expression and cause bacteria to become resistant to FQs (ciprofloxacin) and MEM (Dupont et al., 2005). Ciprofloxacin can cause a decrease in OprN to OprD2 expression, resulting in IMP resistance (Dupont et al., 2005). Additionally, MEM itself can activate the excretion pump system and further cause bacteria to become resistant to various types of drugs. In this study, we found that the patient had used FQs such as ciprofloxacin, levofloxacin, and others during infection. Therefore, the carbapenem resistance that developed during treatment may be associated with the use of FQs medications. FQs medications activate the MexXY-OprM system, resulting in $P$. aeruginosa resistance to carbapenem. Because MEMwas affectived easily the excretion pump resistance mechanism, MEM must be added carefully to the treatment regimen of severely infected patients using FQs medications; these patients must be monitored for sensitivity of $P$. aeruginosa to carbapenem.

According to Liu et al. (2006), single-factor correlation analysis showed that the risk factors of $P$. aeruginosa resistance to carbapenem include old age, chronic obstructive pulmonary disease/bronchiectasis disease, high score in acute physiologic function, and chronic health status (APACHE, mechanical ventilation, simultaneous mixed infection, treatment with FQs medication 15 days before isolation of $P$. aeruginosa, third-generation cephalosporin, and IMP/MEM therapy). Multifactor regression analysis showed that mechanical ventilation and treatment with IMP, or MEM treatment 15 days before isolation of pathogenic bacterium are independent risk factors. In this case, the patient had many of the risk factors described above and a history of IMP therapy; thus, development of $P$. aeruginosa resistance to carbapenem had occurred and may have been related to the host basic state, severity of infection, and use of antibiotics.

\section{ACKNOWLEDGMENTS}

Research supported by the National Natural Science Fund Project (project approval \#81171618). 


\section{REFERENCES}

Chen R, Tang YC and Zhu JX (2001). The research of mechanism in carbapenem resistance of Pseudomonas aeruginosaout membrane protein OprD2 missing of resistance strain. Anti-Infection Chemother. Magazine China 1: 229-230.

Clinical and Laboratory Standards Institute (2005). Performance Standards for Antimicrobial Susceptibility Testing Fifteenth Informational Supplement. M100-S15.

Dupont P, Hocquet D, Jeannot K, Chavanet P, et al. (2005). Bacteriostatic and bactericidal activities of eight fluoroquinolones against MexAB-OprM-overproducing clinical strains of Pseudomonas aeruginosa. J. Antimicrob. Chemother. 55: 518-522.

Kohler T, Michéa-Hamzehpour M, Henze U, Gotoh N, et al. (1997). Characterization of MexE-MexF-OprN, a positively regulated multidrug efflux system of Pseudomonas aeruginosa. Mol. Microbiol. 23: 345-354.

Kolayli F, Karadenizli A, Savli H, Ergen K, et al. (2004). Effect of carbapenems on the transcriptional expression of the oprD, oprM and oprN genes in Pseudomonas aeruginosa. J. Med. Microbiol. 53: 915-920.

Liu HG, Liu J, Xiong SD, et al. (2006). The multidrug resistance states and relative factor analysis of carbapenem resistance or non-resistance with Pseudomonas aeruginosa. Guide Newspaper Med. Ther. 25: 1326-1328.

Luo J, Shen JL, Xu XG, Hu FP, et al. (2007). The research of carbapenem resistance of Acinetobacter -lactamase. Infection Chemother. Magazine China 7: 79-83.

Masuda N, Sakagawa E and Ohya S (1995). Outer membrane proteins responsible for multiple drug resistance in Pseudomonas aeruginosa. Antimicrob. Agents Chemother. 39: 645-649.

Mine T, Morita Y, Kataoka A, Mizushima T, et al. (1999). Expression in Escherichia coli of a new multidrug efflux pump, MexXY, from Pseudomonas aeruginosa. Antimicrob. Agents Chemother. 43: 415-417.

Pfaffl MW (2001). A new mathematical model for relative quantification in real-time PCR. Nucleic Acids Res. 29: e45.

Poole K, Heinrichs DE and Neshat S, (1993). Cloning and sequence analysis of an EnvCD homologue in Pseudomonas aeruginosa: regulation by iron and possible involvement in the secretion of the siderophore pyoverdine. Mol. Microbiol. 10: 529-544.

Quale J, Bratu S, Gupta J and Landman D (2006). Interplay of efflux system, ampC, and oprD expression in carbapenem resistance of Pseudomonas aeruginosa clinical isolates. Antimicrob. Agents Chemother. 50: 1633-1641.

Tenover FC, Arbeit RD, Goering RV, Mickelsen PA, et al. (1995). Interpreting chromosomal DNA restriction patterns produced by pulsed-field gel electrophoresis: criteria for bacterial strain typing. J. Clin. Microbiol. 33: 2233-2239.

Wang F (2006). The monitoring results of bacteria resistance of CHINA in 2005. Infection Chemother. Magazine China 6: 289-295.

Xu HT, Tao FR, Liang YZ, et al. (2007). The research of mechanism in Carbapenem Resistance of Pseudomonas aeruginosa Cli. Infection Chemother. Magazine China 7: 92-95.

Yoneda K, Chikumi H, Murata T, Gotoh N, et al. (2005). Measurement of Pseudomonas aeruginosa multidrug efflux pumps by quantitative real-time polymerase chain reaction. FEMS Microbiol. Lett. 243: 125-131. 